\title{
Efecto de la eliminación de la capa adherente de los huevos utilizando enzima proteolítica proteasa y jugo de piña en la larvicultura del botete diana Sphoeroides annulatus
}

\author{
Effect of eggs desticking using protease proteolytic enzyme and pineapple juice in \\ the larviculture of the puffer fish Sphoeroides annulatus
}

\section{Estela Rodríguez-Ibarra ${ }^{1}$, M. Isabel Abdo-de la Parra ${ }^{1}$, Gabriela Velasco-Blanco ${ }^{1}$, Blanca T. González-Rodríguez ${ }^{1}$, V. Patricia Domínguez-Jiménez ${ }^{1}$, Noemí García-Aguilar ${ }^{1}$ y Leonardo Ibarra-Castro ${ }^{1}$}

\begin{abstract}
'Centro de Investigación en Alimentación y Desarrollo, A.C., Av. Sábalo-Cerritos S/N, C.P. 82000 Mazatlán, Sinaloa, México.eibarra@ciad.mx

Abstract.- Recent findings show that eliminating the gelatinous egg capsule surrounding the fertilized eggs of the puffer fish Sphoeroides annulatus improves embryonic development and hatching rate. In this work, the following 3 different desticking procedures were used to evaluate larval growth and survival: $5 \mathrm{ml} \mathrm{L}^{-1}$ proteolytic protease enzyme (EPP), $1 \%$ of pineapple juice (JP) and eggs with no sticky coating on the surface as a control (C). All treatments were carried out in triplicate and the experiment lasted for 33 days. The fertilization percentage obtained was $98.6 \pm 1.8 \%$ in all treatments. The highest hatching percentage was achieved with EPP $(93.03 \pm 3.01 \%)$ and the lowest with JP $(80.97 \pm 3.97 \%)$. Both total length (LT) $(14.4 \pm 0.40 \mathrm{~mm})$ and weight $(P)(0.118 \pm 0.004 \mathrm{~g})$ were significantly higher in the JP treatment. The highest survival rate was obtained with EPP $(19.34 \pm 1.98 \%)$ and differences were found to be statistically significant. The desticking of eggs treated with JP gave a low hatching percentage due to the fruits $\mathrm{pH}$ reading of 4 and since fewer organisms were produced, higher growth rate was obtained. Thus, we conclude that removing the adhesive layer of the egg does not affect larval growth and survival.
\end{abstract}

Key words: Egg adhesiveness, proteolytic enzyme, pineapple, larvae culture, Sphoeroides annulatus

\section{INTRODUCCIÓN}

Los huevos de los teleósteos se clasifican en demersales y pelágicos, los primeros presentan una hidratación y flotabilidad nula, mientras que los segundos poseen un gran contenido de agua (entre 90 y 95\%), con una flotabilidad positiva en la columna de agua, lo cual facilita un aporte de oxígeno al embrión y su dispersión en el medio marino (Castelló-Orvay 1993, Cerdà 2002).

El botete diana Sphoeroides annulatus (Jenyns, 1842), es una especie con gran demanda y alto precio en el mercado del noroeste de México, ya que su filete es de excelente calidad por su firmeza y su sabor; además es una especie resistente al manejo que se reproduce con facilidad en condiciones de cautiverio (Abdo-de la Parra \& Duncan 2002, Martínez-Palacios et al. 2002, ChávezSánchez et al. 2008, García-Ortega 2009, Rodríguez-Ibarra et al. 2010a). Esta especie forma parte de la fauna acompañante en la pesca del camarón y actualmente se está trabajando en la tecnología e investigación de su cultivo intensivo (Abdo-de la Parra et al. 2010, CONAPESCA 2011). Los huevos de esta especie se clasifican como demersales por las características mencionadas y además poseen una membrana pegajosa, la cual sirve para adherirse durante la etapa embrionaria. En condiciones de cultivo, se ha demostrado que al eliminar esta membrana, los huevos presentan mejores condiciones de incubación, ya que no se aglutinan entre sí, recibiendo una mejor oxigenación y evitando altas mortalidades por anoxia (Al Hazzaa \& Husein 2003, Rodríguez-Ibarra et al. 2010a, b).

Algunos autores señalan la relevancia de usar huevos de peces sin adherencia en la reproducción artificial utilizando diversos métodos, entre ellos: la enzima alcalasa (proteolítica proteasa de acuerdo a Sigma-Aldrich ${ }^{\circledR}$ ), ácido tánico, jugo de piña, leche, talco, arcilla y la técnica clásica de Woynarovich (Billard et al. 1995, Gela et al. 2003, Linhart et al. 2003b, 2004, 2006, Thai \& Ngo 2004, DemskaZakés et al. 2005). En el caso del botete diana, estos métodos fueron aplicados y la enzima proteolítica proteasa y el jugo de piña resultaron ser técnicas simples y rápidas, para eliminar la adherencia de los huevos a los obteniendo un alto porcentaje de eclosión (RodríguezIbarra et al. 2009, 2010a). 
Se ha observado que los huevos del botete diana sin capa adherente presentan una eclosión temprana en comparación con los huevos adherentes y el embrión termina su desarrollo fuera del corion, observando que la larva no presenta pigmentos y movimientos natatorios lentos típicos de una larva recién eclosionada; por lo tanto es necesario evaluar si al eliminar dicha capa adherente afecta la viabilidad de las larvas, al no estar completamente desarrollada podría tener daños físicos e infecciones durante esta etapa (Del Río et al. 2005, Carral et al. 2006). Por lo tanto, el objetivo del presente estudio fue evaluar la eclosión, supervivencia y crecimiento de las larvas de botete diana, utilizando huevos adherentes y sin capa adherente, eliminada con enzima proteolítica proteasa y jugo de piña.

\section{Materiales y MÉTODOS}

Los huevos de botete diana se obtuvieron de una hembra inducida a la maduración por medio de la hormona liberadora de gonadotropina análoga (GnRHa), en implantes tipo EVAc de liberación rápida. Se utilizó semen de un macho maduro sin inducción. Se llevó a cabo la fertilización artificial, mezclando suavemente los huevos junto con el semen y agua marina; posteriormente, se enjuagaron con agua de mar filtrada y esterilizada y se aplicaron los siguientes tratamientos para desgomar los huevos: a) tratamiento con enzima proteolítica proteasa (EPP) Sigma Aldrich ${ }^{\circledR}, 5 \mathrm{ml} \mathrm{L}^{-1}$ durante $8 \mathrm{~min}$; b) tratamiento con jugo de piña (JP) al 1\% durante 3 min y posteriormente eliminación de esta solución, agregando jugo concentrado durante 3 min y c) tratamiento control (C) es decir, huevos sin tratamiento.

\section{INCUBACIÓN}

Cada uno de los tratamientos se aplicó por triplicado y fueron sembrados 6,6 huevos $\mathrm{ml}^{-1}$ en jarras incubadoras McDonald (Aquatic Eco-Systems, Inc.) con capacidad de $6 \mathrm{~L}$. Se evaluó la fertilización a las $3 \mathrm{~h}$ aproximadamente y a las $60 \mathrm{~h}$ la eclosión, utilizando las siguientes fórmulas:

$$
\begin{aligned}
F & =\frac{H_{v}}{H_{v}+H_{m}} * 100 \\
E & =\frac{L}{L+H} * 100
\end{aligned}
$$

donde, $\mathrm{F}=$ porcentaje de fertilización, $\mathrm{Hv}=$ huevos vivos y $\mathrm{Hm}$ = huevos muertos; $\mathrm{E}=$ porcentaje de eclosión, $\mathrm{L}=$ número de larvas y $\mathrm{H}=$ número de huevos.
Para la toma de la muestra de huevos y larvas, se utilizó una pipeta Bogorov (Bogorov \& Zenkevich 1947), con una capacidad de $7 \mathrm{ml}$ (Velasco-Blanco et al. 2011, Rodríguez-Ibarra \& Abdo-de la Parra 2012).

Las larvas de cada uno de los tratamientos fueron transferidas recién eclosionadas a estanques de fibra de vidrio de $600 \mathrm{~L}$ para realizar la larvicultura.

\section{Cultivo larvario}

El cultivo larvario se llevó a cabo a una temperatura promedio de $28,2^{\circ} \mathrm{C}, 5,9 \mathrm{mg} \mathrm{L}^{-1}$ de $\mathrm{O}_{2}$ y salinidad de 32,5 , utilizando el protocolo de Abdo-de la Parra et al. (2011) con algunas modificaciones. El cultivo se realizó usando la técnica de agua verde (García-Ortega 2009, Abdo-de la Parra et al. 2010, Abdo-de la Parra \& Rodríguez-Ibarra 2011), donde a partir del primer día y hasta 13 días después de la eclosión (DDE), a los estanques de cultivo se añadió una vez al día una mezcla de microalgas Nannochloropsis oculata $\left(500,000\right.$ células $\left.\mathrm{ml}^{-1}\right)$ e Isochrysis sp. $(50,000$ células $\left.\mathrm{ml}^{-1}\right)$. Del 2 hasta el 6 DDE se añadieron 10 rotíferos $\mathrm{ml}^{-1}$ (Brachionus rotundiformis) aumentando a 20 rotíferos $\mathrm{ml}^{-1}$ a partir del 7 hasta el $15 \mathrm{DDE}$; y finalmente se redujo a $10 \mathrm{ml}^{-1}$ cuando se añadieron los nauplios de Artemia sp. $\left(0,5 \mathrm{ml}^{-1}\right)$ los cuales se incrementaron gradualmente hasta $4 \mathrm{ml}^{-1}$. El alimento vivo presente en cada estanque se contó diariamente para ajustarlo a la cantidad requerida, la proporción suministrada al día de rotíferos y nauplios de Artemia sp. fue $50 \%$ por la mañana, $25 \%$ a medio día y $25 \%$ por la tarde.

El destete, cambio del alimento vivo al artificial (BuxadéCarbó 1992, García-Ortega 2007), se realizó a partir del 24 DDE, con una dieta comercial microparticulada (Skretting ${ }^{\circledR}$, Fontaine les Vervins, France) reduciendo gradualmente el número de nauplios de Artemia sp. hasta sustituirlos completamente por la dieta artificial.

Las larvas se cultivaron utilizando luz continua las $24 \mathrm{~h}$ durante los primeros 15 DDE. A partir del 16 DDE, el fotoperiodo se redujo a 12:12 h de luz:oscuridad. Del día 1 al 13 DDE se hizo un recambio de agua en un $30 \%$ (aproximadamente $180 \mathrm{~L}$ ) antes de abrirse el flujo, para mantener una buena calidad del agua. A los 14 DDE se suministró flujo continuo haciendo recambios de agua de 0,2 vol día ${ }^{-1}$ y aumentando gradualmente hasta alcanzar 1 a 3 vol día ${ }^{-1}$ al 31 DDE. El flujo de aire se mantuvo a 0,5 $\mathrm{L} \mathrm{min}^{-1}$ del 1 a 15 DDE y se incrementó hasta alcanzar $2 \mathrm{~L}$ $\min ^{-1}$ a partir del $25 \mathrm{DDE}$. 
Durante el cultivo larvario se realizaron biometrías de las larvas, longitud total (LT) y peso (P). Para medirlas se utilizó un microscopio Olympus ${ }^{\circledR}$ utilizando el objetivo $4 \mathrm{X}$ con un micrómetro ocular de $25 \mu \mathrm{m}$ cuando las larvas estaban recién eclosionadas (día 1) y para las mediciones posteriores (10, 18 y $31 \mathrm{DDE})$, se utilizó un vernier digital con una resolución de 0,01 $\pm 0,03 \mathrm{~mm}$. En los días 10 y 18 después de la eclosión (DE), las larvas se pesaron utilizando una balanza digital con una precisión de 0,0001 g, obteniendo el peso promedio de 10 organismos por estanque, ya que debido a que las larvas eran muy pequeñas y la balanza no tuvo precisión para detectar su peso individual, ya para el día $31 \mathrm{DE}$ se pesaron individualmente.

La supervivencia se evaluó al día $33 \mathrm{DE}$, para lo cual se bajó el nivel de agua a un tercio de su capacidad en cada uno de los estanques de cultivo y todos los organismos se capturaron con jarras de plástico y/o redes de cuchara de $10 \mathrm{~cm}$ de nailon fino, y fueron contados en forma individual.

Para determinar la supervivencia se aplicó la siguiente fórmula:

$$
S=\frac{N^{\circ} L f}{N^{\circ} L i} * 100
$$

donde $\mathrm{S}=$ porcentaje de supervivencia, $\mathrm{Lf}=$ número de larvas finales y $\mathrm{Li}=$ número de larvas iniciales.

Los resultados de eclosión y supervivencia fueron transformados a arcoseno; se les determinó la normalidad de su distribución mediante la prueba de Bartlett, y la homocedasticidad de la varianza (prueba de Levene). Tanto los datos de eclosión y supervivencia como los de crecimiento (LT, P), fueron evaluados mediante un análisis de varianza de una vía (ANDEVA, STATGRAPHICS Plus 5.1, Statgraphics ${ }^{\mathrm{TM}}$ net $)(P=0,05)$. Cuando se presentaron diferencias significativas, se analizaron mediante una prueba de contraste múltiple de Tukey $(P<0,05)$ para determinar que medias difieren entre sí.

\section{Resultados y Discusión}

\section{INCUBACIÓN}

El porcentaje promedio de fertilización para los 3 tratamientos fue de $98,6 \pm 1,8 \%$, mientras que el porcentaje de eclosión de los huevos desgomados con EPP fue de $93,03 \pm 3,01 \%$. Para los tratados con JP fue de $80,97 \pm$
$3,97 \%$ y para el C fue de $85,83 \pm 2,78 \%$, presentándose diferencias significativas entre los tres tratamientos $\left(\mathrm{F}_{(2,6)}=10,252, P=0,011\right)$ (Fig. 1).

El uso de la enzima proteolítica proteasa, resultó efectiva en la eliminación de la membrana de los huevos de botete diana (S. annulatus), al igual que lo reportado en las especies dulceacuícolas como la carpa (Cyprinus carpio), tenca (Tinca tinca) y siluro (Silurus glanis), mejorando significativamente el desarrollo embrionario y por consiguiente la eclosión (Gela et al. 2003, Linhart et al. 2003b, 2004, 2006, Rodríguez-Ibarra et al. 2010a). Se ha demostrado en trabajos previos que la eliminación de la membrana adherente de los huevos, es fundamental para el manejo de la reproducción artificial, ya que reduce en su totalidad el efecto pegajoso de la membrana y evita que los huevos se adhieran entre sí, formando masas y provocando altas mortalidades a causa de la anoxia y crecimiento de hongos (Al Hazzaa \& Husein 2003, Walker et al. 2010, Rodríguez-Ibarra et al. 2010a). Además, el uso de esta técnica de desadherencia de los huevos, permite utilizar incubadoras McDonald ${ }^{\circledR}$ como en el presente estudio, ya que el huevo al estar libre de la membrana

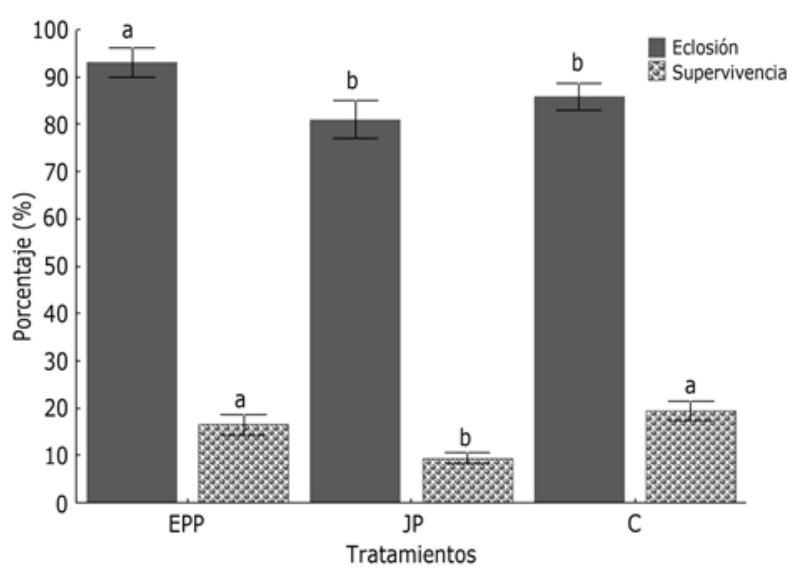

Figura 1. Porcentajes de eclosión y supervivencia de los huevos de botete diana tratados con enzima proteolítica proteasa (EPP) y jugo de piña (JP) y los huevos control (C). Letras diferentes en la barra indican diferencia significativa entre tratamientos $(P<0,05) /$ Hatching and survival rates of puffer fish eggs treated with proteolytic enzyme protease (EPP) and pineapple juice (JP) and eggs as control $(C)$. Different letters in the bar indicate significant differences among treatments $(P<0.05)$ 


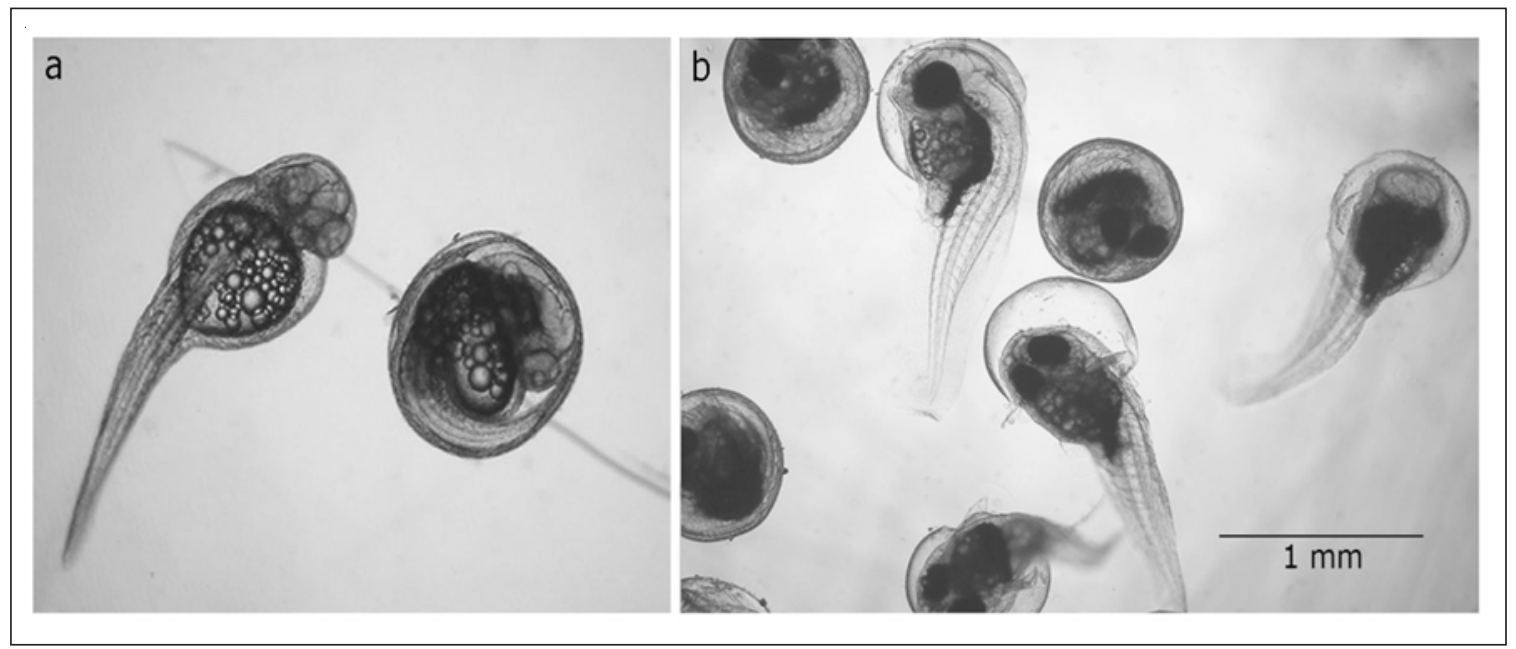

Figura 2. Larvas recién eclosionadas de botete diana Sphoeroides annulatus. a) sin capa adherente y b) con capa adherente / Newly hatched puffer fish Sphoeroides annulatus larvae. a) without sticky outer layer and b) those with sticky outer layer

adherente, permanece en la columna de agua y obtiene una buena oxigenación (Rodríguez-Ibarra et al. 2010b).

En relación al porcentaje de eclosión de huevos tratados con el jugo de piña, existen reportes (Thai \& Ngo 2004, Rodríguez-Ibarra et al. 2009, 2010a) donde los resultados fueron $(86,93$ y $89 \%$, respectivamente). El método aplicado en el presente estudio es similar a los trabajos mencionados, la única variante fue el grado de acidez que presentó la piña ( $\mathrm{pH} 4,0)$, lo cual pudo afectar el desarrollo embrionario. Este efecto de la acidez, se ha observado en especies como la perca y carpa (Rask 1984, Oyen et al. 1991), donde un pH menor a 5 afectó a los huevos en etapas tempranas de desarrollo y a las larvas recién eclosionadas de dichas especies. El uso de un producto natural como el jugo de la piña, por una parte es una alternativa para eliminar la capa adherente de los huevos y mejorar su incubación, con la ventaja que es económica y accesible durante todo el año en la región noroeste de México, donde se cultiva el botete diana (Rodríguez-Ibarra et al. 2010a), pero tiene la desventaja que al momento de usarse presenta acidez que afecta el desarrollo embrionario, como sucedería con los embriones del botete diana en el presente estudio.

En el caso de los huevos del tratamiento control, se obtuvieron porcentajes de eclosión muy superiores $(85,83$ $\pm 2,78 \%$ ) al reportado por Rodríguez-Ibarra et al. (2010a) que fue de $18,82 \pm 5,39 \%$, incubados en estanques de fibra de vidrio de $600 \mathrm{~L}$. En el presente estudio, los huevos incubados en jarras McDonald $\AA$, aunque tendieron a aglomerarse entre sí por la capa adhesiva que poseen, recibieron una óptima oxigenación en este sistema de incubación, porque la mayor parte del tiempo permanecieron flotando en la columna de agua por el efecto de la aireación.

El periodo de incubación de los huevos del botete diana fue de 96 h y la eclosión inicia a las 72 h (GarcíaOrtega et al. 2003), pero al eliminar la capa adherente el tiempo es menor y en promedio la eclosión inicia a las 60 $\mathrm{h}$ a una temperatura entre 25 y $26^{\circ} \mathrm{C}$ (Rodríguez-Ibarra et al. 2010a, b). Al evaluar las larvas recién eclosionadas de los huevos sin adherencia y adherentes, se observó que todas las larvas de los primeros tenían poco movimiento y carecieron de pigmentación en los ojos, y algunas presentaron un notocordo encorvado (Fig. 2). Estas mismas características de las larvas fueron descritas por Abdo-de la Parra et al. (2012), en huevos de botete diana incubados a una temperatura de $31{ }^{\circ} \mathrm{C}$, los cuales eclosionaron $48 \mathrm{~h}$ después de la fertilización. Carral et al. (2006), observaron que al eliminar la capa adherente a los huevos de la tenca (Tinca tinca), presentaron una eclosión prematura y los consideraron embriones, entre las fases de la segmentación caudal y la pigmentación de los ojos. Estas mismas características se presentaron en los huevos de botete diana desgomados con la enzima proteolítica proteasa y con el jugo de piña, ya que al eliminarse la capa adherente, el corion queda más delgado y esto permite a la larva romperlo más fácilmente y adelantar su eclosión (Rodríguez-Ibarra et al. 2010a) 


\section{Cultivo larvario}

Las larvas al eclosionar midieron en promedio de LT 2,03 $\pm 0,03,2,01 \pm 0,09$ y $1,98 \pm 0,04 \mathrm{~mm}$ para los tratamientos con EPP, JP y C, respectivamente, sin diferencias significativas entre ellos, lo cual indica que no hubo efecto en su tamaño al eliminar la capa adherente en comparación con las larvas sin tratamiento, resultado que coincide con Rodríguez-Ibarra et al. (2010a) (Tabla 1).

El cultivo larvario tuvo una duración de 33 días, iniciando el destete de los peces a más temprana edad (día 24 DE) a diferencia del trabajo de Abdo-de la Parra et al. (2011), quien llevó el cultivo larvario a los 45 días DE e iniciando el destete al día $30 \mathrm{DE}$. De acuerdo al análisis de varianza (ANDEVA), aplicado a los datos de supervivencia, se encontraron diferencias significativas entre los tratamientos $\left(\mathrm{F}_{(2,6)}=21,791, P=0,001\right)$, donde el promedio más alto se obtuvo con los huevos tratados con EPP (19,34 $\pm 1,98 \%)$, seguido de los huevos adherentes (C) $(16,44 \pm 2.28 \%)$ y el promedio más bajo resultó en los huevos tratados con JP $(9,43 \pm 1,25 \%)$ (Fig. 1). En el presente trabajo se obtuvo una supervivencia superior a la obtenida por Abdo-de la Parra et al. (2011), quienes reportaron $12,5 \%$, lo cual puede atribuirse a que en el presente estudio se hizo un recambio de agua del $30 \%$ desde el inicio del cultivo, a diferencia de un recambio de agua $100 \mathrm{~L}$ (16\%) realizado por Abdo-de la Parra et al. (2011). Esta variable pudo contribuir a mantener una buena calidad de agua en los estanques de cultivo y un mayor desarrollo de las larvas.

Las larvas cultivadas con el tratamiento con JP, fueron más grandes en LT $(14,4 \pm 0,40 \mathrm{~mm})$ como en $\mathrm{P}(0,118 \pm$ $0,004 \mathrm{~g}$ ), que de acuerdo al análisis de varianza (ANDEVA) resultaron estadísticamente diferentes a las larvas de los otros 2 tratamientos $\left(\mathrm{F}_{(2,6)}=21,767, P=0,001 \mathrm{y} \mathrm{F}_{(2,6)}=\right.$ $38,236, P=0,000$ ) (Tabla 1). Las larvas del tratamiento con JP crecieron más que las de los otros 2 tratamientos, ya que la cantidad de individuos fue menor y tuvieron una mayor disponibilidad de alimento y espacio.

La mayoría de las investigaciones realizadas sobre la eliminación de la capa adherente en huevos, utilizando la enzima proteasa y/o el jugo de piña, se han llevado a cabo en especies dulceacuícolas y sólo han reportado resultados hasta la eclosión (Linhart et al. 2000, 2003a, b, Gela et al. 2003, Thai \& Ngo 2004). Sin embargo, el único trabajo que ha reportado resultados desde la fertilización hasta el cultivo larvario es el de Carral et al. (2006). Dichos autores probaron 4 tratamientos (2 dosis de enzima alcalasa, ácido tánico y solución Woynarovich) para
Tabla 1. Resultados de longitud total inicial y final y peso final (promedio \pm desviación estándar) de larvas de Sphoeroides annulatus de los 3 tratamientos al final del cultivo larvario (33 días). Valores promedio con superíndices diferentes indican diferencias significativas / Results of initial and final total length and final weight (mean \pm standard deviation) of all 3 treatments for Sphoeroides annulatus at the end of the culture experiment $(33$ days). Mean values with different superscripts indicate significant differences

\begin{tabular}{cccc} 
Tratamientos & $\begin{array}{c}\text { Longitud total } \\
\text { inicial }(\mathrm{mm})\end{array}$ & $\begin{array}{c}\text { Longitud total } \\
\text { final }(\mathrm{mm})\end{array}$ & $\begin{array}{c}\text { Peso } \\
(\mathrm{g})\end{array}$ \\
\hline EPP & $2,03 \pm 0,03$ & $10,8 \pm 1,31^{\mathrm{a}}$ & $0,056 \pm 0,014^{\mathrm{a}}$ \\
JP & $2,01 \pm 0,09$ & $14,4 \pm 0,40^{\mathrm{b}}$ & $0,118 \pm 0,004^{\mathrm{b}}$ \\
C & $1,98 \pm 0,04$ & $9,95 \pm 0,65^{\mathrm{a}}$ & $0,045 \pm 0,012^{\mathrm{a}}$
\end{tabular}

eliminar la adherencia de los huevos de la tenca (Tinca tinca). Ellos mencionan la eclosión prematura de las larvas debido al efecto de la desadherencia de los huevos y describen a la larva como embriones ya que mostraban esas características señalando que su desarrollo embrionario es finalizado en el exterior del corion. La hipótesis que plantearon fue que los embriones podían presentar daños físicos o infectarse durante la incubación a altas densidades; procedieron a realizar un segundo experimento donde determinaron que al adelantar la eclosión de los huevos desadherentes, esto no influye en la supervivencia y crecimiento de las larvas. Esta misma característica se presentó en el botete diana, donde también se observó que no hubo efectos negativos en el desarrollo y crecimiento de las larvas de esta especie.

Se concluye que eliminar la capa adherente de los huevos de S. annulatus, no afectó el crecimiento y supervivencia de las larvas. Además se determinó que la optimización de los protocolos de cultivo del botete diana, favorece la eclosión, obteniendo porcentajes de supervivencia muy superiores a los publicados en trabajos anteriores. Por lo tanto, se recomienda el uso de la enzima proteolítica proteasa para eliminar la adherencia de los huevos y para el cultivo larvario del botete diana.

\section{Agradecimientos}

Los autores agradecen a V. Williams de Calvario por la traducción del resumen y la asistencia técnica de J.F. Arias-Rodríguez, J. Huerta, R. Mendoza y J. Tirado. 


\section{LITERATURA CITADA}

Abdo-de la Parra MI \& NJ Duncan. 2002. Avances en cultivo experimental de botete diana (Sphoeroides annulatus). Panorama Acuícola 7(2): 42-43.

Abdo-de la Parra MI \& LE Rodríguez-Ibarra. 2011. Cultivo larvario y requerimientos nutricionales del pargo flamenco, 63 pp. LAP LAMBERT Academic Publishing Gmbh \& Co. KG, Saarbrücken.

Abdo-de la Parra MI, A García-Ortega, I MartínezRodríguez, B González-Rodríguez, G Velasco-Blanco, C Hernández \& N Duncan. 2010. An intensive hatchery rearing protocol for larvae of the bullseye puffer, Sphoeroides annulatus (Jenyns). Aquaculture Research 41(10): 554-560.

Abdo-de la Parra MI, LE Rodríguez-Ibarra, G VelascoBlanco, N García-Aguilar, JA Ibarra-Soto, NJ Duncan \& LS Álvarez-Lajonchère. 2011. Cultivo larvario del botete diana (Sphoeroides annulatus) en tres diferentes densidades de siembra. En: Ruiz-Luna A, CA BerlangaRobles \& M Betancourt-Lozano (eds). Avances en acuicultura y manejo Ambiental, pp. 93-106. Editorial Trillas, México.

Abdo-de la Parra MI, IE Martínez-Rodríguez, BT González-Rodríguez, LE Rodríguez-Ibarra, N Duncan \& C Hernández-González. 2012. Efecto de la temperatura y salinidad del agua en la incubación de huevos de botete diana Sphoeroides annulatus. Revista de Biología Marina y Oceanografía 47(1): 147-153.

Al Hazzaa R \& A Hussein. 2003. Stickiness elimination of himri barbel (Barbus lutes, Heckel) eggs. Turkish Journal of Fisheries and Aquatic Sciences 3: 47-50.

Billard R, J Cosson, G Perchec \& O Linhart. 1995. Biology of sperm and artificial reproduction in carp. Aquaculture 129: 95-112.

Bogorov VG \& LA Zenkevich. 1947. Instructions for carrying out hydrobiologic work in the sea (plankton and benthos), 126 pp. Glavservovpati, Moscow.

Buxadé-Carbó C. 1992. Zootecnia: bases de producción animal. Producción Animal Acuática 13: 1-377. Ediciones Mundi Prensa, Madrid.

Carral JM, JD Celada, M Sáenz-Royuela, R Rodríguez, A Aguilera \& P Melendre. 2006. Effects of four egg desticking procedures on hatching rate and further survival and growth of larvae in the tench (Tinca tinca L.). Aquaculture 37: 632-636.

Castelló-Orvay F. 1993. Acuicultura marina: Fundamentos biológicos y tecnología de la producción. Ciències Experimentals i Mtemàtiques, 739 pp. Universitat de Barcelona, Barcelona.

Cerdà J. 2002. Mecanismos fisiológicos durante la hidratación del huevo de teleósteos: hacia el desarrollo de nuevos métodos de criopreservación. Boletín Instituto Español de Oceanografía 18(1-4): 145-152.
Chávez-Sánchez MC, LS Álvarez-Lajonchère, MI Abdode la Parra \& N García-Aguilar. 2008. Advances in the culture of the Mexican bullseye puffer fish Sphoeroides annulatus, Jenyns (1842). Aquaculture Research 39: 718730 .

CONAPESCA. 2011. Anuario estadístico de acuacultura y pesca, 305 pp, Comisión Nacional de Acuacultura y Pesca, México. <http://www.conapesca.sagarpa.gob.mx/wb/cona/ cona_anuario_estadistico_de_pesca>

Del Río V, J Rosas, A Velásquez \& T Cabre. 2005. Desarrollo embrionario-larval y tiempo de metamorfosis del pez tropical Xenomelaniris brasiliensis (Pisces: Atherinidae). Revista de Biología Tropical 53(3-4): 503-513.

Demska-Zakés K, Z Zakés \& J Roszuk. 2005. The use of tannic acid to remove adhesiveness from pikeperch, Sander lucioperca, eggs. Aquaculture 36: 1458-1464.

García-Ortega A. 2007. Estado actual de la investigación y producción de botete diana Sphoeroides annulatus. En: González-Félix ML, L Bringas-Alvarado, M PérezVelázquez \& M Meza-García (eds). Memorias del 3er Foro Internacional de Acuicultura, 28-30 de noviembre, Sonora México, pp. 58-83.

García-Ortega A. 2009. Nutrition and feeding research in the spotted rose snapper (Lutjanus guttatus) and bullseye puffer (Sphoeroides annulatus), new species for marine aquaculture. Fish Physiology and Biochemistry 35(1): 6980 .

García-Ortega A, I Abdo \& C Hernández. 2003. Weaning of bullseye puffer (Sphoeroides annulatus) from live food to microparticulate diets made with decapsulated cysts of Artemia and fishmeal. Aquaculture International 11: 183194.

Gela D, O Linhart, M Flajshans \& M Rodina. 2003. Egg incubation time and hatching success in tench Tinca tinca (L.) related to the procedure of egg stickiness elimination. Journal of Applied Ichthyology 19: 132-133.

Linhart O, D Gela, M Flajshans, P Duda, M Rodina \& V Novák. 2000. Alcalase enzyme treatment for elimination of egg stickiness in tench, Tinca tinca L. Aquaculture 191: 303-308.

Linhart O, D Gela, M Flajshans \& M Rodina. 2003a. Proteolytic enzyme treatment: an improved method for elimination of egg stickiness in tench, Tinca tinca L., in aquaculture. Journal of Applied Ichthyology 19: 134-137.

Linhart O, M Rodina, D Gela, M Flajshans \& M Kocour. 2003b. Enzyme treatment for elimination of egg stickiness in tench (Tinca Tinca L.), European catfish (Silurus Glanis L.) and common carp (Cyprinus carpio L.). Fish Physiology and Biochemistry 28: 507-508.

Linhart O, D Gela, M Rodina \& M Kocour. 2004. Optimization of artificial propagation in European catfish, Silurus glanis L. Aquaculture 235: 619-632. 
Linhart O, M Rodina, M Kocour \& D Gela. 2006 Insemination, fertilization and management in tench, Tinca tinca (L.). Aquaculture 14: 61-73.

Martínez-Palacios CA, MC Chávez-Sánchez, GS Papp, MI Abdo-de la Parra \& LG Ross. 2002. Observations on spawning early development and growth of the puffer fish Sphoeroides annulatus (Jenyns, 1843). Journal of Aquaculture in the Tropics 17: 59-66.

Oyen FGF, LECMM Camps \& SE Wendelaar -Bonga. 1991. Effects of acid stress on the embryonic development of the common carp (Cyprinus carpio). Aquatic Toxicology 19: $1-12$.

Rask M. 1984. The effect of low $\mathrm{pH}$ on perch, Perca fluviatilis L. II. The effect of acid stress on different development stages of perch. Annales Zoologici Fennici 21: 9-13.

Rodríguez-Ibarra LE \& MI Abdo-de la Parra. 2012. Desgomado, desinfección e incubación de huevos de botete diana: Optimización de métodos para la obtención de larvas de botete diana (Sphoeroides annulatus), 64 pp. LAP LAMBERT Academic Publishing Gmbh \& Co, Saarbrücken.

Rodríguez-Ibarra LE, GA Rodríguez-Montes de Oca, CY Padilla-Aguiar, VP Domínguez-Jiménez, MA SánchezRodríguez, G Velasco-Blanco \& N García-Aguilar. 2009. Aplicación de productos naturales y químicos para desgomar los huevos de botete diana Sphoeroides annulatus. Industria Acuícola 5(6): 13-15.
Rodríguez-Ibarra LE, MI Abdo-de la Parra, GA RodríguezMontes de Oca, MS Moreno-Hernández, G VelascoBlanco, N García-Aguilar \& LS Álvarez-Lajonchère. 2010a. Evaluación de métodos para la eliminación de la capa adherente de los huevos del botete diana Sphoeroides annulatus (Jenyns, 1842). Revista de Biología Marina y Oceanografía 45(1): 147-151.

Rodríguez-Ibarra LE, GA Rodríguez-Montes De Oca, CY Padilla-Aguilar, VY Zepeda-Mercado \& G VelascoBlanco. 2010b. Evaluación de dos métodos de incubación de huevos de botete diana Sphoeroides annulatus. Industria Acuícola 6(5): 4-7.

Thai BT \& TG Ngo. 2004. Use of pineapple juice for elimination of egg stickiness of common carp (Cyprinus carpio L.). Asian Fisheries Science 17: 159-162.

Velasco-Blanco G, AC Puello-Cruz, LS ÁlvarezLajonchere, BT González-Rodríguez, MI Abdo-de la Parra, LE Rodríguez-Ibarra \& A García-Ortega. 2011. Alimento vivo. En: Álvarez-Lajonchère LS \& AC PuelloCruz. (eds). El pargo flamenco: Lutjanus guttatus. Producción controlada de huevos, larvas y juveniles, pp. 59-97. Clave Editorial, México.

Walker AB, D Ward, K Duclos, M Peters \& DL Berlinsky. 2010. Surface disinfection and removal of adhesiveness from rainbow smelt eggs. North American Journal of Aquaculture 72: 158-163. 